\title{
When Ion or Electron Channeling Meets Crystal Orientation Mapping
}

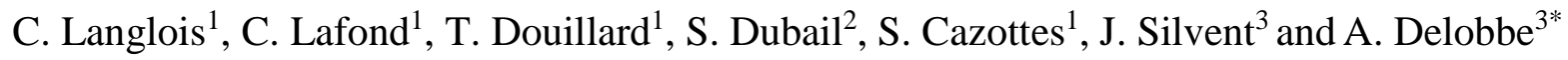 \\ 1. MATEIS laboratory - INSA Lyon, Lyon, France. \\ 2. Axon Square Ltd, Sciez, France. \\ 3. Tescan Orsay Holding, Fuveau, France. \\ * Corresponding author: anne.delobbe@ orsayphysics.com
}

It is well known that Electron Back Scattered Diffraction (EBSD) method is an efficient technic to obtain orientation maps. Nevertheless, like every analysis means, it has some drawbacks and we would like to present an alternative called Channeling Orientation Determination (CHORD) [1,2] based on channeling contrast. This channeling contrast information is obtained either with an electron or ion image series. The polycrystalline sample is pre-inclined with respect to the beam and an image is taken while rotating the sample (Figure $1 \mathrm{a})$ ). Along such image series, each $(\mathrm{X}, \mathrm{Y})$ pixel of the region of interest undergoes an intensity variation due to the channeling effect, that can be plotted as a function of the rotation angle. Such intensity profiles can be theoretically predicted for a given orientation of a crystal. The indexation procedure then relies on a search in a database of theoretical profiles obtained by simulating intensity profiles for a large set of orientations.

In the case of primary ion beam such as in a FIB (Focused Ion beam), iCHORD image series are obtained by ion-induced secondary electrons detection. An intensity loss is observed when the ion beam arrives parallel to some low index crystallographic planes of a crystal. Therefore, if the atomic structure of the crystal is projected onto a surface perpendicular to the ion beam, an intensity loss will correspond to large "free spaces" between the atomic projections, which are quantified by summing the grey levels of the projection pixels. An efficient model of the channeling effect is then obtained following this principle (Figure 1b)), provided precautions to avoid projection artefacts.

For back scattered electron image series, the similarity between the eCHORD and Electron Channeling Patterns (ECP) acquisitions is used to model quantitatively the experimental electron channeling effect. The eCHORD intensity profiles are then simulated by extracting the intensity along a circle from simulated ECPs [3].

In the resulting orientation maps, the angular resolutions are both under $1^{\circ}$, with slightly better performances using electrons (around $0.3^{\circ}$ ) compared to ions $\left(0.9^{\circ}\right)$. More generally, the main advantage is that no extra detector is needed to carry out the experiment, opening orientation mapping capability potentially on any SEM and FIB machine. Moreover, acquisition times are comparable to EBSD technique. The geometry of acquisition, simpler than the EBSD one, could be also a critical advantage when turning to 3D orientation mapping.

In this paper, we would like to show the interest of CHORD in the case of microelectronics samples. Innovative semiconductor devices are composed of a variety of materials in structures of high complexity, inevitably leading to mechanical stresses inside the device potentially detrimental to the performance or even reliability of the product. Designing dependable and durable microelectronic products therefore requires techniques to accurately measure the intrinsic stresses. Particularly, orientation and strain mapping is a reliable method to highlight some mechanical disorders. 
Because it can be easily implemented on any SEM and FIB, CHORD can be the right choice for addressing several microstructural issues typical from microelectronics. Without being exhaustive, we can mention: copper lines microstructure after dual damascene, Die attach material characterization, especially $\mathrm{PbSnAg}$ or $\mathrm{SnAgCu}$ (microstructure, grain size, texture, intermetallic nature). The interface between front side metallization and bonding wire (respectively aluminum and gold materials) can also be characterized finely by CHORD in terms of grain size and texture as a function of the deposition technique.

In this presentation, pros and cons of CHORD approach compared to EBSD will be discussed. We will focus on samples from microelectronics and elements like copper and aluminum (figure 2). The simplicity of the implementation will be demonstrated. Indeed, the sample preparation which consists in cross section and polishing of the region of interest, is done directly in the FIB-SEM instrument and assess a better process, minimizing the stress on the samples.

\section{References :}

[1] C. Langlois et al., Ultramicroscopy 157 (2015) p. 65.

[2] C. Langlois et al., Ultramicroscopy 186 (2018) p. 146.

[3] S. Singh and M. De Graef, Microscopy \& Microanalysis 23 (2017) p. 1.

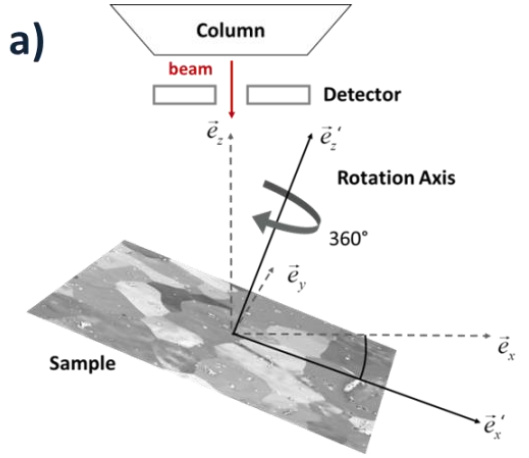

b)

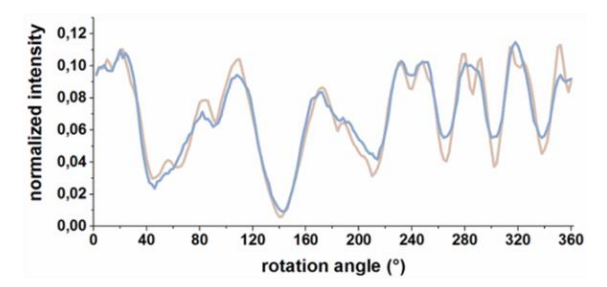

FIGURE 1. a) CHORD setup b) Concordance between experimental (blue) and theoretical (brown) intensity profiles.
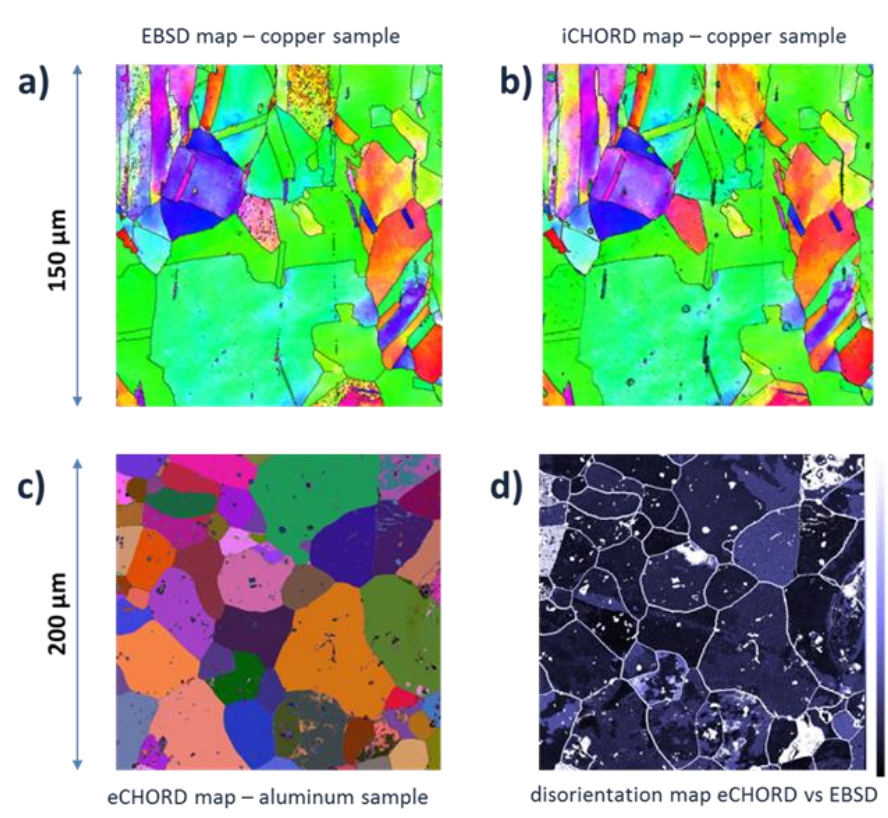

d)

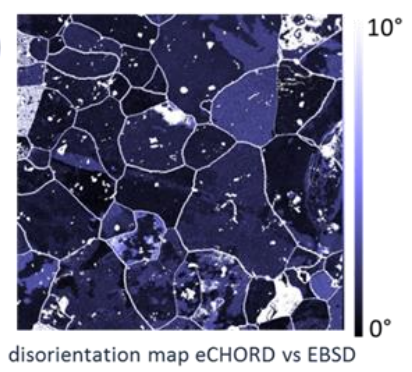

FIGURE 2. a) EBSD map on a copper sample, b) iCHORD map on the same area, c) eCHORD map on an aluminum sample, $d$ ) disorientation map eCHORD vs EBSD on the same area. 